\title{
EMMLi: A maximum likelihood approach to the analysis of modularity
}

\begin{tabular}{|r|l|}
\hline Journal: & Evolution \\
\hline Manuscript ID & Draft \\
\hline Manuscript Type: & Brief Communication \\
\hline Date Submitted by the Author: & $\mathrm{n} / \mathrm{a}$ \\
\hline Keywote List of Authors: & $\begin{array}{l}\text { Goswami, Anjali; University College London, Genetics, Evolution \& } \\
\text { Environment } \\
\text { Finarelli, John; University College Dublin, School of Biology \& } \\
\text { Environmental Science }\end{array}$ \\
\hline & $\begin{array}{l}\text { Morphological Evolution, phenotypic integration, trait correlations, } \\
\text { mammals, model selection }\end{array}$ \\
\hline
\end{tabular}


1 EMMLi: A maximum likelihood approach to the analysis of modularity

2 Running Header: Maximum likelihood analysis of modularity

4 Anjali Goswami ${ }^{1}$ and John A. Finarelli ${ }^{2,3}$

5

$6{ }^{1}$ Department of Genetics, Evolution \& Environment and Department of Earth Sciences,

7 University College London, London, WC1E 6BT, UK

$8{ }^{2}$ School of Biology \& Environment Science, University College Dublin, Science Centre - West,

$9 \quad$ Belfield, Dublin 4, Ireland.

$10{ }^{3}$ UCD Earth Institute, University of College Dublin, Belfield, Dublin 4, Ireland.

11 Emails: a.goswami@ucl.ac.uk; john.finarelli@ucd.ie

12

13 Keywords: phenotypic integration, trait correlations, mammals, model selection

14 
Identification of phenotypic modules, semi-autonomous sets of highly-correlated traits, can be

17 accomplished through exploratory (e.g., cluster analysis) or confirmatory approaches (e.g., RV

18 coefficient analysis). While statistically more robust, confirmatory approaches are generally unable to compare across different model structures. For example, RV coefficient analysis finds support for both two- and six-module models for the therian mammalian skull. Here, we present a maximum likelihood approach that takes into account model parameterization. We compare model log-likelihoods of trait correlation matrices using the finite-sample corrected Akaike

23 Information Criterion, allowing for comparison of hypotheses across different model structures.

24 Simulations varying model complexity and within- and between-module contrast demonstrate 25 that this method correctly identifies model structure and parameters across a wide range of conditions. We further analyzed a dataset of 3-D data, consisting of 61 landmarks from 181

27 macaque (Macaca fuscata) skulls, distributed among five age categories, testing 31 models, 28 including no modularity among the landmarks, and various partitions of 2, 3, 6, and 8 modules.

29 Our results clearly support a complex six-module model, with separate within- and inter-module 30 correlations. Furthermore, this model was selected for all five age categories, demonstrating that 31 this complex pattern of integration in the macaque skull appears early and is highly conserved 32 throughout postnatal ontogeny. Subsampling analyses demonstrate that this method is robust to relatively low sample sizes, as is commonly encountered in rare or extinct taxa. This new

34 approach allows for the direct comparison of models with different parameterizations, providing 35 an important tool for the analysis of modularity across diverse systems. 


\section{INTRODUCTION}

38 The related topics of phenotypic integration and modularity, which concern associations among

39 traits and their partitioning into semi-autonomous and highly-correlated subsets, respectively,

40 have received increased attention over the past few decades as a powerful bridge among different

41 scales of evolutionary analysis. Recent years have seen increasing effort to identify and compare

42 phenotypic modularity and integration across taxa, in some cases spanning entire vertebrate

43 'classes' (Goswami 2006b, a; Goswami 2007; Porto et al. 2009; Bell et al. 2011; Bennett and

44 Goswami 2011; Klingenberg and Marugan-Lobon 2013), and even comparing plants and animals

45 (Conner et al. 2014). There has also been a refining of different levels of modularity acting at

46 different scales. The most typically-studied level, termed "variational" (Marquez 2008) or

47 "static" (Klingenberg 2014) modularity, focuses on a single species or population, commonly at

48 a specific ontogenetic stage (e.g., adults). Within this level, analyses focus on identifying drivers

49 of trait integration, whether functional, developmental, genetic, or environmental. Beyond

50 variational modularity, studies have analyzed modularity at the ontogenetic scale (that is,

51 patterns or changes in modularity through ontogeny within a species), and evolutionary

52 modularity (comparative analysis of patterns of modularity across taxa). Coincident with this

53 increase in studies of modularity, there has been an explosion in the number of methods

54 proposed to analyze phenotypic modularity and integration, both within and across populations

55 (Klingenberg 2009; Goswami and Polly 2010; Klingenberg 2013; Adams and Felice 2014;

56 Bookstein and Mitteroecker 2014; Klingenberg 2014). 
58 Analyses of modularity have taken many forms, from entirely exploratory approaches, such as

59 cluster analysis, Euclidean distance matrix analysis, and graphical modelling, to confirmatory

60 approaches, such as partial least squares analysis and the related RV coefficient analysis,

61 integration matrices, and theoretical matrix modelling (reviewed in Klingenberg 2009; Goswami

62 and Polly 2010; Klingenberg 2013, 2014), and there has been a vigorous discussion of the merits,

63 practical considerations, and issues of each approach (Klingenberg 2008; Goswami and Polly

64 2010; Fruciano et al. 2013; Adams and Felice 2014). Not surprisingly, confirmatory methods are

65 generally viewed as more robust, particularly as exploratory methods such as cluster analysis

66 impose hierarchical relationships on traits that may or may not reflect their true biological

67 organization. On the other hand, exploratory approaches have the benefit of not requiring $a$

68 priori determination of model structure, whereas confirmatory methods depend on a defined

69 model structure and are therefore limited to testing pre-selected models. Given the complexity of

70 many biological structures, and the diverse factors that may influence trait relationships

71 (Hallgrimsson et al. 2009), this limitation argues for the continued role of exploratory

72 approaches, particularly as studies expand beyond well-established model systems. Recent work

73 has developed relative eigenanalysis for the purpose of comparing two covariance matrices in a

74 more informative manner than do previous methods, such as eigenvalue dispersion or random

75 skewers analysis (Bookstein and Mitteroecker 2014), providing an efficient exploratory approach

76 that can detail the specific ways that high-dimensional covariance matrices differ by identifying

77 the maximal ratios of variance between any two groups. However, this approach does not

78 directly address the problem of describing the pattern of integration for a group, which remains

79 an outstanding issue in this field. 
81 Another important issue with most current confirmatory approaches is that they are designed to

82 measure support for alternative hypothesized parameter values within a proposed model structure

83 (Wagner 2000). For example, RV coefficient analysis determines the correlations among sets of

84 traits, and then randomizes trait associations to produce an empirical distribution of RV

85 coefficients for the model structure under consideration, testing the hypothesis that the observed

86 RV coefficient is significantly lower than randomized alternatives. But while this methodology

87 can test if a particular model is more structured than random, it does not readily address the

88 question of whether a four-module model describes the pattern of phenotypic integration better

89 than arrangements with three or five modules. The same is true of the recently described

90 Covariance Ratio metric (Adams 2016), which improves upon several statistical issues with RV

91 coefficient analysis, but also can only test one model of modularity against a hypothesis of

92 random associations of traits. Thus far, only one published method allows for comparisons of

93 models with different complexities (Marquez 2008), as demonstrated with a 2-D landmark

94 dataset for rodent mandibles. This method included several innovations that allowed for testing

95 of hundreds of alternative models, including those with overlapping landmarks, but the most

96 relevant is the correction of similarity among the observed and modeled covariance matrices

97 against the number of estimated parameters. This addition facilitates comparison across models

98 with varying structures of different complexity. While this represented an important step in

99 confirmatory tests of modularity, the author noted that a linear correction for the number of

100 estimated parameters may not be appropriate for all test statistics or for more complex

101 approaches (Marquez 2008). Additionally, this method has also never been expanded to 3-D

102 data. 
104 Here, we describe a new method for the analysis of phenotypic modularity from trait correlation 105 matrices based on maximum likelihood approach. We provide a case study applying this 106 approach to a dataset of macaque skulls spanning infant to adult age groups. We use this method 107 to compare various models that have been proposed for mammalian skull modularity (including 108 no modularity, a two-module neurocranial/facial hypothesis, and multiple six-module

109 hypotheses; Fig. 1), as well as novel alternative models of varying structure and complexity.

112 Model selection approaches using information theory compare likelihood fits across a set of 113 models of varying degree of complexity. In order to estimate likelihoods of models of trait 114 integrations, we first model the expected distribution around a hypothesized value representing 115 the relationship among a set of traits. For the product moment correlation coefficient, and its 116 derivatives including the congruence coefficient and canonical correlation (Goswami and Polly 117 2010), a simple transformation is available in the Fisher r-to-z transformation:

119 Eq. 1) $z_{r}=\tanh ^{-1}(\boldsymbol{r})=\frac{1}{\mathbf{2}} \ln \left(\frac{\mathbf{1}+\boldsymbol{r}}{1-\boldsymbol{r}}\right)($ Sokal and Rohlf 1995, pg. 575),

121 where $r$ is the sample correlation coefficient. Here the observed correlation matrix is treated as a 122 set of realizations (the values of $r$ ) of a hypothesized true correlation coefficient $(\rho)$. The 
123 distribution around a hypothesized value of $\rho$ is approximately normally distributed with 124 parameters:

125

126 Eq. 2a) $\boldsymbol{\mu}_{\rho}=z_{\rho}=\frac{1}{2} \ln \left(\frac{1+\rho}{1-\rho}\right)$ and,

127 Eq. 2b) $\boldsymbol{\sigma}_{\boldsymbol{\rho}}{ }^{2}=\left(\frac{1}{\sqrt{n-3}}\right)^{2}=\frac{1}{n-3}($ Sokal and Rohlf 1995, page 575),

129 where $\mathrm{n}$ is the sample size used to calculate the correlation coefficient (i.e., the number of

130 specimens with measured landmarks). The log-likelihood support for a hypothesized value of $\rho$, 131 given an observed value of $r$, is then:

133 Eq. 3) $\log \boldsymbol{L} \propto-\frac{\mathbf{1}}{\mathbf{2}} \boldsymbol{L n}\left(\boldsymbol{\sigma}_{\boldsymbol{\rho}}{ }^{2}\right)-\frac{\left(\boldsymbol{z}_{\boldsymbol{r}}-\boldsymbol{\mu}_{\boldsymbol{\rho}}\right)^{2}}{{\mathbf{2 \sigma _ { \rho }}}^{2}}($ Edwards 1992). modularity) proposes a single value for the correlation coefficient between all possible trait pairs.

137 The value that maximizes the summed log-likelihood for all observed correlations in the matrix 138 would then be the preferred hypothesis, and this log-likelihood would then be the model log139 likelihood for the "no modularity" model structure. 
141 However, given the results of a large number of previous studies (Cheverud 1982, 1989, 1995a, 142 1996; Ackermann and Cheverud 2000; Marroig and Cheverud 2001; Hallgrimsson et al. 2004;

143 Goswami 2006a; Hallgrimsson et al. 2009; Porto et al. 2009; Goswami and Polly 2010;

144 Klingenberg 2013), it is highly likely that a model structure positing a single value of $\rho$ for the 145 entire correlation matrix would not adequately describe trait correlations in a real biological 146 system. Model structures of varying complexity can be compared using the Akaike Information 147 Criterion (AIC) (Akaike 1973; Burnham and Anderson 2002), assessing the likelihood fit of the 148 models, while controlling for better fit induced by increased model complexity. The finite149 sample $\mathrm{AIC}\left(\mathrm{AIC}_{\mathrm{c}}\right)$ is given by:

151 Eq. 4) $\boldsymbol{A I} \boldsymbol{C}_{\boldsymbol{c}}=-\mathbf{2} \boldsymbol{L o g} \boldsymbol{L}+\mathbf{2} \boldsymbol{K}+\frac{\mathbf{2} \boldsymbol{K}(\boldsymbol{K}+\mathbf{1})}{\boldsymbol{N}-\boldsymbol{K}-\mathbf{1}}$ (Hurvich and Tsai 1989).

153 In Equation 4, $\mathrm{N}$ is the sample size, but in the case of computing $\mathrm{AIC}_{\mathrm{c}}$, this is the number of 154 between-trait correlations used to calculate the likelihood score. $\mathrm{K}$ is the number of estimated 155 parameters, which is the number of distinct, optimal correlations estimated by the model, and an 156 additional parameter for each estimate of the variance around the hypothetical value of $\rho$ (see: 157 Equation 2 b). In the present analysis, this is fixed for all of the examined models within each 158 data set (a single variance was calculated for each data set based on its sample size), and the 159 number of parameters is simply the number of estimated values of $\rho$ incremented by one for all 160 models. However, this does not need to be the case, as more complex analyses may wish to 161 consider whether patterns of modularity are common across multiple data sets which may have 
162 different estimates of variance. In such cases, different variances may be included as estimated 163 parameters among different models.

165 To illustrate the designation of model parameters more clearly, consider a set of landmarks 166 across a mammal cranium (Fig. 2A). Previous study of the mammal skull has proposed six 167 modules for this system (Cheverud 1982; Goswami 2006a). It is possible that that the 168 magnitudes of within-module correlations are effectively the same in all of the modules (Fig. 2B) 169 or that each of these modules has distinct strengths of correlation between landmarks within a 170 given module (Fig. 2C). Furthermore, inter-module correlations could also be distinct for each 171 module-to-module set (Fig. 2E and G), or they could be effectively identical (Fig. 2D and F).

172 These variations then returns four potential model structures with 3, 17, 8 or 22 estimated 173 parameters (the number of estimated $\rho$ 's in each, plus 1 for the estimated variance). Summing the 174 log-likelihoods from Equation 3 for the set of observed correlations within each modeled set for 175 an optimal estimate of $\rho$, gives the model log-likelihood. These can be compared to one another, 176 to the "no modularity" hypothesis, and to different proposed structures or different groupings of

177 the landmarks within modules using Equation 4. From the model $\mathrm{AIC}_{\mathrm{c}}$ scores, we calculate $178 \Delta \mathrm{AIC}_{\mathrm{c}}$, the difference between a particular model's $\mathrm{AIC}_{\mathrm{c}}$ score and the lowest score observed 179 among the tested models. From this, we calculate the model log-likelihood adjusting for the 180 penalty due to parameterization:

182 Eq. 5) Model Log $\boldsymbol{M} \propto-\frac{\mathbf{1}}{\mathbf{2}} \Delta \boldsymbol{A} \boldsymbol{I} \boldsymbol{C}_{\boldsymbol{c}}$ (Burnham and Anderson 2002). 
184 A set of model posterior probabilities can then be calculated by dividing each model's likelihood 185 by the sum of likelihoods over the set of examined models (N.B. these are likelihoods, and are

186 therefore equal to $\mathrm{e}^{\text {Model LogL }}$ (see: Burnham and Anderson 2004)).

A value of " $n$ " or sample size appears in both the equations for calculating the variance around an estimated value of $\rho$ (Equation 2b) and for the calculation of the AIC statistic (Equation 5).

191 We have used upper- and lowercase to distinguish between the two, as $n$ for calculation of 192 correlations is based on the number of specimens, whereas, in the case of computing $\mathrm{AIC}_{\mathrm{c}}, N$ is 193 the number of between-trait correlations considered in calculating the log-likelihood. For a 61 194 landmark data matrix, there are 1830 unique between-landmark correlations (i.e., the sub195 diagonal values of the matrix).

The Fisher r-to-z Transformation converts the bounded correlation coefficient to an unbounded variable. Comparison of the transformed correlation to a hypothetical population value of $\rho$ demonstrates that the transformed coefficient is approximately normally distributed about $\rho$, 201 making the Fisher Transformation attractive for hypothesis testing. In the case of the correlation matrix, however, there is a concern about the independence of the sample of correlation 
203 coefficients, in that, for example, elements $r_{12}$ and $r_{13}$ are not strictly random iid draws from a 204 population, but are themselves intercorrelated. However, the Fisher-transformed correlations 205 within a correlation matrix have been shown to be asymptotically, multivariate normal in 206 distribution, and robust to the violations of independence (Steiger 1980b; De Leeuw 1983).

207 Specifically, this has been demonstrated for pattern hypotheses within correlation matrices, 208 wherein observed correlation coefficients are tested against a proposed "pattern matrix" (Steiger 209 1980a), and this approach, which is adopted here in the form of the proposed within- and among210 module correlation estimates, has been applied in a wide range of research questions (Feldman et 211 al. 2007; Wager et al. 2007; LeBel and Gawronski 2009). As such the employing Fisher212 transformed correlations in a likelihood framework, as proposed here, should prove a reliable 213 approach to evaluating modularity with trait correlation matrices.

\section{SIMULATIONS}

216 Given the above noted concern with respect to independence of the Fisher-transformed

217 correlation coefficients, we evaluated the ability of the maximum likelihood approach as

218 implemented in EMMLi to correctly select a known model when choosing among models

219 structures. To do so, we conducted an extensive series of simulations testing a range of model

220 structures, contrasting two variables: model complexity (number of parameters) and contrast

221 (difference between within-module and between-module strength of integration). In all cases, 60

222 "landmarks" were simulated as divided into zero, two or six modules, to represent a hypothetical

223 correlation structure that we wish to evaluate. Between-module correlations were set at a mean

224 value of 0.1 for all simulations. Standard deviations for generating correlations were varied from 
225 a low value of $\sigma=0.01$ to realistic value of $\sigma=0.05$ (e.g., Cheverud 1982), encompassing values

226 used in simulations testing other recently described methods for the analysis of modularity 227 (Adams 2016).

Simulating datasets without any modular structure allowed for assessment of Type I error rates. 100 permutations each were run with the mean correlations among all traits simulated as $r=$ $0.15,0.3,0.5,0.7$, or 0.9 , with $\sigma=0.01$ or 0.05 , for a total of 1000 simulations. In these cases, the correct model would be equivalent in structure to model $1(\mathrm{~K}=2)$ in Table 1.

For the two and six module structures, both simple and complex models were tested. The simple models involved two or six modules which all had the same within-module correlations, set to

236 five mean values ranging from $r=0.15$ in the lowest contrast model to $r=0.9$ in the highest

237 contrast model (i.e., mean within-module $r=0.15,0.3,0.5,0.7$, and 0.9 were all simulated).

239 For the complex models, all two or six modules had different within-module correlations. In the 240 high contrast, complex two-module model, these values were set to mean within-module $r=0.7$ 241 and 0.9 ; in the mix contrast model, mean within-module $r=0.3$ and 0.8 ; and in the low contrast 242 case, mean within-module $r=0.15$ and 0.3 . In the high contrast, complex six-module model, 243 mean within-module $r=0.7,0.75,0.8,0.85,0.9$, and 0.95 ; in the mix contrast case, mean within244 module $r=0.3,0.4,0.5,0.6,0.7$, and 0.8 ; and in the low contrast case, mean within-module $r=$ $2450.15,0.2,0.25,0.3,0.35$, and 0.4 . For the simple two-module structure, the correct model would 
246 be equivalent in structure to model $2(\mathrm{~K}=3)$ in Table 1 , and the complex structure would be

247 equivalent to model $3(\mathrm{~K}=4)$. For the simple six-module structure, the correct model would be

248 equivalent in structure to model 4 or $8(\mathrm{~K}=3)$ in Table 1 , and the complex structure would be

249 equivalent to model 5 or $9(\mathrm{~K}=8) .100$ permutations each of these 16 models were run, using

250 each of the standard deviation levels, resulting in 3200 total simulations of these modular

251 structures.

252

253

254 CASE STUDY: MAXIMUM LIKELIHOOD ANALYSIS OF MACAQUE CRANIAL

255 MODULARITY

256 Materials

257 We use a data set of 3-D coordinates for 61 landmarks taken on the cranium of Japanese

258 macaque (Macaca fuscata) from the Primate Research Institute at Inuyama, Japan, previously

259 described in (Goswami and Polly 2010) (see Supporting Information). Individuals were divided

260 into five datasets representing four age classes: infants with deciduous dentition only $(\mathrm{n}=42)$,

261 juveniles with M1 erupted $(n=42)$, sub-adult with M2 erupted $(n=48)$, and adults with the

262 entire adult dentition, further divided into male and female data partitions $\left(\mathrm{n}_{\mathrm{m}}=25, \mathrm{n}_{\mathrm{f}}=24\right)$. See

263 Goswami and Polly (2010) for further details on the dataset used in the following analyses. 
265 The landmark data were superimposed with Generalized Procrustes superimposition to remove

266 the effects of rotation, translation and size (scaling all specimens to unit centroid size). All five

267 datasets were analyzed separately. We calculated vector congruence coefficient correlation

268 matrices, producing $61 \times 61$ element matrices. This vector-based approach allows for

269 simultaneous analysis of all three coordinates representing a single landmark (Goswami 2006a;

270 Goswami and Polly 2010). There has been some debate about the use of vector-based versus

271 coordinate-based correlations in studies of phenotypic integration and modularity (Klingenberg

272 2008; Goswami and Polly 2010; Klingenberg 2013). Here, we use the vector-based matrices, as

273 we feel these better reflects biological relationships, treating each landmark as a single unit of

274 information. However, we also include an example using the correlation matrix for individual

275 coordinates for the M1-erupted data set (see Supporting Information). This is a 183x183 matrix

276 (x-, y- and z-coordinates for each of 61 landmarks). Allometric effects and asymmetric variation

277 have not been removed from the example dataset, for comparability with previously published

278 analyses of macaque skull modularity (Cheverud 1982; Goswami and Polly 2010), although, as

279 with selection of metric of trait correlation, the model presented here is applicable to datasets that

280 do remove, or focus entirely on, those aspects of shape.

282 Models

283 We investigated 31 model structures within several broad hypotheses of cranial modularity. The

284 first, and simplest, model structure is that there are no distinct modules within the cranium, and

285 that the cranium can be analyzed as a single entity. Further, more complex, models of modularity

286 consist of a two-module (neurocranial vs. facial) structure (Drake and Klingenberg 2010), two 
287 six-module structures (primate-specific (Cheverud 1995b) and general mammalian (Goswami 288 2006a)), and an eight-module structure combining the two six-module models (see: Table S1).

289 We investigated further refinements for both configurations of the six-module structure: first, 290 leaving some landmarks "unintegrated", i.e., outside of any module, based on a monotreme 291 model of integration (Goswami 2006a), resulting in 3-module + "unintegrated" models; and, 292 second, considering a tissue-origin model (Goswami 2006a), in which landmarks were grouped 293 based on their derivation from neural crest, mesodermal, or mixed germ-layer derived bone (see: 294 Table S1).

As detailed above, each hypothesized model structure may have many potential

297 parametrizations, depending on whether within-module or across-module correlations are 298 modeled as being the same for all cases (e.g., a single high hypothesized correlation within 299 modules and a single, across-module correlation), or all module cases are considered unique, or 300 some mixture of these extremes. For example, the 2-module neurocranial/facial model structure comprises Models 2 and 3 (Table 1), with the difference being the number of proposed within-

302 module estimates. Models with increasing numbers of modules have correspondingly greater 303 complexity in their potential parameterizations. As described above, the six-module model has 304 four different parameterizations examined here (Fig. 2). In the simplest model (Model 4, Fig. 305 2D), there is a single within-module estimate and a single across-module estimate. Other models 306 propose six freely-varying within-module estimates with a constant across-module estimate 307 (Model 5, Fig. 2F), fifteen freely-varying across-module estimates with a single within-module estimate (Model 6, Fig 2E) and a completely varying model with six within-module estimates and 15 across-module estimates (Model 7, Fig. 2G). All model structures that were explored and 
310 their corresponding parameterizations are given in Table 1 . The R code used in this analysis and

311 example data files are provided in the online supporting information for this article and are

312 available for download from: http://www.goswamilab.com/\#!software/c1cxq.

\section{Subsampling analysis}

315 While analyses of integration are often performed on model systems with the ability to sample

316 large numbers of individuals, questions about the evolution of integration can require the

317 incorporation of fossil or rare taxa (Goswami et al. 2015) for which sample sizes are constrained.

318 To evaluate potential sensitivity of this method to small sample sizes, we conducted a

319 subsampling analysis of the best sampled dataset (subadult Macaca, 48 specimens), producing

32050 random subsets each of 25 specimens, 15 specimens, and 10 specimens. Each subset was

321 subjected to generalized Procrustes analysis prior to calculation of vector congruence coefficient

322 correlation matrices, producing 61x61 element matrices and analyzed in EMMLi.

\section{$324 \quad$ RESULTS}

\section{Simulations}

326 When a low standard deviation $(\sigma=0.01)$ around the simulated correlation values was used, the

327 correct model structure was identified as the best fit model in $100 \%$ of cases for all no-module,

328 two-module, and six-module structures (Fig. 3A). Reconstructed $\rho$ values were consistently

329 within 0.01 of the simulated values. For the simulations of a no-modularity data set, posterior 
330 probabilities were generally low, $\sim 0.24$, even for the best fit model. All posterior probabilities

331 for the correct model were greater than 0.5 for the simulations in which there was a modular

332 structure to the data. In all cases, estimated $\rho$ values exactly matched those used to generate the

333 simulated datasets.

When a higher standard deviation of 0.05 was used, the correct model was identified in most cases, although accuracy decreased at the highest levels of mean correlations for simple structures (Fig. 3B). The correct model was selected with high $(>0.90)$ posterior probability in $100 \%$ of cases for the simple six-module model with within-module correlations ranging from 0.15 to 0.70 . It was also correct, with $100 \%$ posterior probability, in all cases for the complex 340 six-module structure, using either high, mixed, or low correlations. When all within-module correlations were set to 0.90 , the correct model was selected in $23 / 100$ runs, and receives a

342 posterior probability $>0.05$ in $36 / 100$ runs, with a different parameterization of the same model 343 structure ( 344 correct model was selected in $100 \%$ of cases for within-module correlations of $0.15,0.30$, and 345 0.50. The correct model is selected in $84 / 100$ cases when the within-module correlation is 0.7 , 346 and receives a posterior probability $>0.05$ in $100 \%$ of cases. In the remaining 16 runs, the 347 closely related, more parameterized two-model model $(\mathrm{K}=3)$ was selected as the best fit model. 348 When within-module correlations are centered around 0.90 , an unrelated model was selected in 349 the majority of cases. The correct model was selected in $100 \%$ of cases with the complex two350 module model using low or mixed correlations. When only the highest correlations $(0.70$ and 351 0.90) were used to simulate a complex two-module structure, the correct model was selected in $35277 / 100$ cases and had a posterior probability $>0.05$ in 83/100 cases. 
354 The strongest effects of high correlations and higher standard deviation were observed in cases

355 of no modularity in the simulated structure (Fig. 3B). The correct model was selected in $100 \%$

356 of cases when the overall correlation was 0.15 or 0.30 . When the overall correlation was 0.50 ,

357 the correct model was selected as the best fit model in 98/100 runs and had a posterior

358 probability $>0.05$ in all runs. With overall correlations of 0.70 , the correct model was selected 359 as the best fit model in 53/100 cases and had a posterior probability $>0.05$ in 95 cases. In the

360 cases where the wrong model was selected, the posterior probability was $<0.50$ in all but five

361 cases, although, as noted above, posterior probabilities are generally low $(\sim 0.2)$ for models of no

362 modularity, even when the correct model was selected. When the overall correlation was

363 extremely high, 0.90 , the wrong model was selected with posterior probability $>0.50$ in all runs.

364 Even in cases where the wrong model was supported, estimated $\rho$ values were within 0.03 of the

365 values used to simulate each dataset.

367 Case study

368 For all five data sets, the optimal model selected by $\mathrm{AIC}_{\mathrm{c}}$ was Model 7 (Fig. 1C), with over 99\%

369 of the posterior probability centered on this model for each data set, with the remaining model

370 posterior probabilities were effectively zero for all other models considered (Tables 2, S2-S5).

371 Additionally, the $183 \times 183$ raw coordinate data the juvenile (M1 erupted) data set (Table S6) also

372 returned Model 7 as the unambiguously best-supported model. Model 7 can thus be considered

373 the single optimal model describing the pattern of cranial integration in the macaque data set

374 (Edwards 1992; Royall 1997; Burnham and Anderson 2002). 
376 Model 7 is based on Cheverud's primate-specific six-module structure (Cheverud 1982),

377 proposing distinct within-module $\rho$ 's for all six modules, as well as separate $\rho$ 's for all possible

378 across-module comparisons (total of 22 estimated parameters). Model 16, for the adult female

379 data set only, had a posterior probability of $\sim 0.001$ (Table S2). This model is a variant of Model

3807 , in which the oral, nasal, and occipital modules are maintained, but all other landmarks are 381 treated as unintegrated, which is broadly similar to the pattern of modularity displayed by

382 monotremes (Goswami 2006a). All other model structures, including those that proposed no

383 modularity, a neurocranial/facial module structure, more than six cranial modules, or non-

384 primate specific module structures, received no support.

386 Estimated values for $\rho$ were similar for each of the 21 model parameters across the four data sets

387 (Table 3), with very strongly integrated anterior modules (Modules 1 and 2, corresponding to the 388 anterior dentition and nasal/facial bones) and a moderately integrated occipital region (Module 389 6). Other modules, corresponding to the basicranium, neurocranium, and palatal $/$ molar region 390 were less well integrated, as were inter-module correlations. This is in approximate agreement 391 with previous analyses of integration patterns in mammalian crania (Goswami 2006a).

394 For the subsampling analyses, the unambiguously best supported model (posterior probabilities $>$ 395 0.95) was the same as for the full dataset (Model 7) 100\% of the time, for the rarefaction to 25 
specimens. With 15 specimens, the same model was selected in 48/50 analyses. In the two cases

397 of mismatch, Model 7 was one of three top models (posterior probability $>0.05$ ), sharing support

398 with alternative parameterizations of the same Cheverud six-module structure. Subsampling to

39910 specimens recovered Model 7 in 36/50 of runs. In three of the remaining runs in which it

400 wasn't the best fit model, it was selected as one of the top models ( $>0.05$ posterior probability),

401 in all cases along with alternative parameterizations of the Cheverud six-module structure. For

40211 runs, Model 7 had a posterior probability less than 0.05 . Thus, even at $n=10$, this method was

403 successful at identifying the correct model as having a significant posterior probability $78 \%$ of

404 the time. Moreoever, of the 14 cases where Model 7 was not the top model, the best supported

405 model was a variation on the Cheverud model in 12 cases. In only 2 of the 50 runs was the top

406 model unrelated to Model 7; thus, a relevant model structure, if not the correct parameterization, 407 was recovered in $96 \%$ of cases at $n=10$.

409 Reconstructed $\rho$ values were consistently very similar to those of the full dataset (Table 4), even

410 at $n=10$, with mean deviations from $\rho$ values for the full dataset of 0.020 for $n=25$, to 0.037 for

$411 \mathrm{n}=15$, and 0.062 for $\mathrm{n}=10$. Standard deviations of reconstructed $\rho$ values were similarly low,

412 but unsurprisingly increasing with decreasing sample sizes: 0.023 for $n=25,0.036$ for $n=15$,

413 and 0.042 for $n=10$. Thus, these further analyses provide strong support that this method is

414 remarkably robust to quite low sample sizes. 


\section{DISCUSSION}

419 Extensive simulations varying model complexity, magnitude of mean within-module correlation, 420 and standard deviation of correlations demonstrates that this method is robust under biologically

421 realistic conditions. It performs exceedingly well (perfectly, in fact), when correlations are

422 tightly grouped around hypothetical values of $\rho$ (low standard deviation simulations), regardless

423 of whether the simulated structure is highly modular or entirely lacks any modular structure.

424 With increased dispersion around the $\rho$ values (higher standard deviations), this method is robust 425 under most conditions, but struggles with highly integrated structures, specifically those that 426 combine two biologically unlikely situations: 1) complete lack of modularity and 2) uniformly 427 and, in most cases, unrealistically high correlations. Only in the case of very high within-module 428 correlations (mainly $\rho=0.90$, but also involving $\rho=0.70$ in the no-modularity model and in the 429 high-correlation complex two-module model) does the method return incorrect model structures with high posterior probability. Observing such high correlations, uniformly across all modules

431 or an entire structure is unusual. Previous studies (Conner et al. 2014) have shown that

432 vertebrates, plants, and hemimetabolous insects display mean phenotypic correlations among 433 linear traits ranging from 0.35 to 0.5 , although mean correlations among linear traits in 434 holometabolous insects may be much higher ( 0.84). In the case study presented here, only a 435 single module (Module 2) shows mean within-module correlations above 0.7 (Table 3), while all 436 other modules are in the moderate to low range of within-module correlations used in these

437 simulations. Our simulations also show that this method is extremely robust in identifying 438 complex models of modularity in which some modules have high within-module correlations and 439 others have moderate or low within-module correlations. Thus, outside of the unusual conditions 
440 noted above, our method proves to work with high efficacy, and the few cases of "failure" in

441 conditions typically encountered in most biological systems involved selection of a differently

442 parameterized version of the same model structure.

444 We further note that no other method currently available for confirmatory analysis of modularity

445 directly compares models of modularity against a model of total integration (e.g., Marquez 2008;

446 Klingenberg 2009; Adams 2016). For example, in the description of the covariance ratio metric,

447 the author provided the important cautionary note that covariance ratio analysis be used only for

448 evaluating patterns of modularity and suggested that Partial Least Squares analysis (Rohlf and

449 Corti 2000; Adams and Felice 2014) be used to evaluate hypotheses of integration (Adams

450 2016). EMMLi thus provides unprecedented ability to evaluate models of total integration as

451 well as models of modularity, but struggles with correctly identifying the lack of modularity

452 when both standard deviations of correlations and mean correlations are high. For this reason,

453 we urge caution in interpreting results if the returned posterior probabilities of the best fit models

454 are low $(<0.50)$, if reconstructed correlations are exceptionally high (uniformly $>0.70$ ), or if

455 multiple unrelated models are returned with posterior probability $>0.05$, particularly if standard

456 deviations of within-module correlations are high. Under those circumstances, we follow Adams

457 (2016) in suggesting that it may prove useful to employ Partial Least Squares analysis to

458 evaluate the support for a highly integrated structure. We further advise users to consider and

459 report all models with posterior probabilities greater than 0.05 . 
461 With regard to the macaque case study, for all five data sets, greater than $99 \%$ of the posterior 462 probability distribution was explained by Model 7, the most parameterized version of

463 Cheverud's model of six cranial modules. This result indicates very strong support for this model 464 of cranial modularity in macaques. Cheverud's (1982) model structure was based on analysis of 465 correlations among inter-landmark distances (length measurements) from a dataset of 462 rhesus 466 macaques (Macaca mulatta). Cheverud (1982) identified support for this model by calculating an 467 agreement statistic between the hypothesized F-sets and empirical P-sets, the latter derived by 468 cluster analysis of inter-landmark distances in principal component space. This model structure 469 has subsequently tested using theoretical matrix correlation analysis and RV coefficient analysis, 470 with the present Japanese macaque dataset (M. fuscata) (Goswami and Polly 2010). However, 471 that study also tested two alternative models: the two-module facial/neurocranial model (Models 472 2-3 in Table 1), and an alternative six-module structure (the "Goswami" models, Models 8-11 in 473 Table 1), based on general patterns of integration among therian mammals (Goswami 2006a). In 474 that study, model selection was not directly possible, as RV coefficient analysis makes no 475 specific hypothesis regarding model parameterization beyond the total number of modules and 476 theoretical matrix correlation analysis simply compares the correspondence between two 477 matrices, usually with a permutation test to assess support. All three model structures were 478 supported at $\mathrm{p}<0.01$ using theoretical matrix correlation analysis with Mantel's test, although it 479 should be noted that Cheverud's model showed the highest correlations with the empirical data. 480 In the RV coefficient analyses, the two-module model was supported in three of the five datasets $481(\mathrm{p}<0.05)$, the Goswami model was supported in two of five datasets, and the Cheverud model 482 supported in three of the five datasets, and, where supported, the Cheverud model received the 
483 strongest support $(\mathrm{p}<0.001)$. However, it was not supported for either adult dataset, whereas

484 both the two-module and the Goswami models received support for the adult male dataset.

486 The Goswami and Polly (2010) analysis highlighted an important issue with the existing range of

487 confirmatory approaches to analyzing modularity: the lack of a clear way to compare among 488 models across proposing fundamentally different structures of modularity/integration. One can 489 compare the Cheverud six-module model to the Goswami six-module model with RV coefficient 490 analysis, as they both are based on six cranial modules, yet neither can be meaningfully 491 compared to the two-module neurocranial/facial model (Fig. 1). Moreover, there are a range of 492 possibilities, from unintegrated traits within a partially modular structure, to entirely different 493 modular structures that are biologically interesting and potentially informative, but which are 494 impossible to approach with the existing methods.

496 The results presented demonstrate the unambiguous support for Cheverud's structure of 497 phenotypic modularity for the macaque cranium, with distinct within- and among-model 498 correlation values. Here, we used maximum likelihood analysis of congruence coefficients 499 derived from multidimensional vector variables, as well as the more standard individual 500 coordinate correlations for one dataset. We focused on trait correlation matrices, rather than 501 variance-covariance matrices, in this method, as the relationships among traits, and not their 502 individual variances, are the primary concern in studies of phenotypic integration and modularity 503 (Olson and Miller 1951; Olson and Miller 1958; Pavlicev et al. 2009; Goswami and Polly 2010; 504 Conner et al. 2014). Benefits of the model selection approach employed here include: 1) ability 
505 to directly compare models of different complexities (such as two- and six-module models) or

506 models of similar complexity which do not constitute nested subsets of one another (such as the

507 Cheverud (1982) and Goswami (2006a) six-module models), 2) increased precision in model

508 description, in terms of varying numbers of within- and between-module values for $\rho$; and 3)

509 expansion to mixed models, in which a structure can include both modules and unintegrated

510 traits (e.g., models 20-31 in Table 1).

512 As noted above, there is an existing method to compare competing models of variational

513 modularity using subspace analysis (Marquez 2008). As with the maximum likelihood approach

514 described here, subspace analysis is a remarkably flexible approach that accurately reflects the

515 complexity of biological systems and is capable of comparing hundreds of models (and indeed

516 performs better with more models).

518 Both subspace analysis and EMMLI can test multiple variations on a basic model structure, 519 allow for combined or overlapping modules, and conduct direct comparison of models with 520 similar or different parametrizations. In contrast to maximum likelihood analysis as implemented

521 in EMMLi, subspace analysis creates a specific hypothetical covariance matrix for each matrix

522 that fixes between-module covariances at zero. This is rarely the case in biological systems,

523 particularly in proximal modules, and therefore oversimplifies the apparent hierarchical pattern

524 of modularity in systems such as the cranium. The maximum likelihood-based approach

525 described here could be considered preferable because it does not assign an a priori value to 526 between-module correlations, and by returning all estimated $\rho$ values for the best supported 
527 model(s), allows for direct assessment of every within- and between-module correlation, which

528 can inform on alternative model structures to test (for example, if two modules show a between-

529 module $\rho$ that is equal or similar to their respective within-module $\rho$ values, one could add an

530 additional model that unites those modules into a single grouping).

532 The two methods also differ on the method of model selection. As a measure of goodness of fit

533 between the observed and model covariance matrices, subspace analysis as implemented in

534 MINT (Marquez 2008) uses $\gamma$, and corrects for differences in the parametrizations of each model

535 by regressing $\gamma$ against the number of zero elements in each model, generating $\gamma^{*}$, with

536 significance evaluated against expectations from random covariance matrices. In order to

537 strengthen the evaluation of model rank, a jackknifing approach was used, with model support

538 reflecting how often a model ranked first in the jackknifed samples. The method described here

539 does not require fixing any values, but instead provides an overall model structure and searches

540 for values of $\rho$ that return the maximum likelihood for that structure. The complexity of the

541 model, and correction for the goodness of fit or model selection, is a function of the number of

542 independent estimates of $\rho$, rather than the number of zero elements in the model.

544 Because subspace analysis as implemented in MINT has never been developed for 3-D data, we

545 did not conduct a direct comparison of these two methods. Qualitative comparison of the

546 simulations of subspace analysis (Marquez 2008) and those described here suggest that the

547 maximum likelihood approach is more robust to sample size, number of models, model

548 complexity, and magnitude of integration, as well as being available for use with any 
549 morphometric dataset. Nonetheless, subspace analysis represented a major improvement on

550 existing methods, and there are numerous interesting aspects to subspace analysis as

551 implemented in MINT, such as the heuristic modeling of additional hypotheses of modularity

552 and the construction of consensus models, both of which could be developed as exploratory tools 553 within a likelihood framework.

555 In addition to the possibility of incorporating aspects of the Marquez (2008) method, which was 556 developed for the same purpose as the maximum likelihood method described here, there is also 557 vast potential for combining with methods developed for different goals. For example, the 558 Reimmanian spaces for covariance matrices and the distances therein provide a framework for 559 comparing the relative likelihood of one covariance matrix to that of another (Bookstein and 560 Mitteroecker 2014) and could be combined with the method we describe here. In whatever combination, all of these methods are beginning to fill an important need for approaches that are

562 more flexible to the biological reality of complex anatomy.

564 These benefits are important, as many studies of phenotypic modularity to date have either 565 assumed a hypothesized set of modules without explicitly testing its validity for the taxon of 566 interest (e.g., applying the Cheverud model to other mammals, as in Marroig et al. 2009; Porto

567 et al. 2009), or have tested a single model in the absence of comparison to other potential 568 models, regardless of the support for that one model (e.g., Klingenberg and Marugan-Lobon 569 2013). Ongoing analyses of other groups suggest that the Cheverud model does not adequately 570 describe all mammalian taxa. For example, EMMLi analysis of a 55 landmark data set for the red 
571 fox, Vulpes vulpes (Table S7) recovered the 22-parameter version of the Goswami six-module

572 model as the unambiguous best fit model (for details of dataset, see Goswami 2006b). This result

573 is perhaps unsurprising, as that model was initially based on cluster analyses of a comparative

574 dataset that included a large sample of carnivorans (Goswami 2006a). However, it underscores

575 the flexibility of the model selection approach advocated here, in that many different proposed

576 model structures can be simultaneously compared. The approach implemented in EMMLi, and

577 its many possible future extensions, provides the ability to directly compare diverse hypotheses

578 on the evolution of modularity and integration, which will become increasingly crucial as we

579 drift further from well-established model systems. Further work along these lines will be crucial 580 to identifying where shifts in modularity occur in the tree of life, and what the consequences of 581 those shifts may be for the morphological evolution.

With respect to cranial modularity in macaques, the results from maximum likelihood analyses

584 as implemented in EMMLi underscore two important biological points: 1) the model of two 585 cranial modules based on a neurocranial and a facial module is not supported when compared 586 with more complex six-module hypotheses, and 2) the 8-module structure, although biologically 587 plausible, is not supported. This implies that while a functional model of a facial (masticatory) 588 vs. neurocranial organization of the skull is too simplistic to describe phenotypic integration, 589 there is also likely an upper limit to the complexity of cranial integration in the macaque system. 590 In addition, because Model 7 is highly-supported in the infant, juvenile, and subadult data sets in 591 addition to the two adult data sets, this pattern of morphological integration appears to be 592 established very early in postnatal ontogeny in Macaca. This consistency through ontogeny 593 confirms the previous analyses of this dataset (Goswami and Polly 2010), which suggested that, 
594 although relative level of integration decreases through ontogeny, the overall pattern is

595 conserved from infancy to adulthood.

596

597 CONCLUSIONS

598 The study of phenotypic modularity has seen rapid growth in recent years. New empirical studies 599 are expanding the topic beyond model systems through development (Young 1959; Zelditch 600 1988; Hallgrimsson et al. 2004; Zelditch et al. 2006; Goswami et al. 2009; Hallgrimsson et al. 601 2009; Zelditch et al. 2009; Sears et al. 2012), across the tree of life (Armbruster et al. 2004;

602 Young and Hallgrimsson 2005; Goswami 2006b, a; Goswami 2007; Bell et al. 2011; Bennett and 603 Goswami 2011; Armbruster et al. 2014; Conner et al. 2014; Goswami et al. 2014), and even into 604 the distant past (Goswami 2006a; Bell et al. 2011; Gerber and Hopkins 2011; Webster and 605 Zelditch 2011a, b; Maxwell and Dececchi 2012; Meloro and Slater 2012; Gerber 2013; Goswami 606 et al. 2015). Alongside this extension of taxonomic and temporal sampling, there has been an 607 expansion of analytical tools for the evaluation of modularity and integration. Confirmatory 608 approaches, in particular, have received much attention in recent years, with RV coefficient 609 analysis in particular being heavily applied to the analysis of modularity. However, these 610 approaches by and large are limited to the direct comparison of models with similar complexities 611 and do not allow for mixed models, where some traits are highly integrated and others are not.

612 The issues caused by these weaknesses in the existing approaches will become increasing 613 problematic as workers diverge from well-studied models into new systems without well614 established a priori hypotheses of trait relationships. 
616 Here, we have presented a maximum likelihood and model selection approach to the evaluation

617 of modularity, which can directly compare highly complex hypotheses of trait relationships,

618 including comparisons of nested and non-nested models. We demonstrate this approach using

619 multidimensional vector correlation matrices for a large dataset of macaque crania, confirming

620 the results of previous analyses, but allowing, for the first time, robust discrimination of

621 alternative models. Our results support a highly parameterized model of six cranial modules, 622 with distinct levels of integration within modules, as well as between pairs of modules. This

623 method is applicable to any metric of trait relationship, given the availability of an appropriate

624 transformation, has appropriate Type I error rates, is robust to low sample sizes, and should be 625 incorporated into the existing toolbox for the study of phenotypic modularity in diverse systems.

\section{ACKNOWLEDGEMENTS}

628 We thank Prabu Sivasubramaniam and Tim Lucas for development of the R code for EMMLi.

629 Data were gathered at the Primate Research Institute in Inuyama, Japan, with funding provided 630 by the Japanese Society for the Promotion of Science HOPE grant to AG. We thank M. Takai for 631 his role in encouraging and facilitating the research trip to PRI. We thank D. Adams, P.D. Polly, 632 E. Sherratt, C. Klingenberg, UCL's ADaPTiVE lab group, and the NHM-UCL-IC Palaeobiology 633 journal club for relevant discussions and comments on this work. The development of this 634 method was supported by a Leverhulme Trust research grant to AG (RPG 2013-124) and a 635 European Research Council grant (ERC-STG-2014-637171) to AG. 


\section{REFERENCES}

Ackermann, R. R. and J. M. Cheverud. 2000. Phenotypic covariance structure in tamarins (genus Saguinus): a comparison of variation patterns using matrix correlation and common principal components analysis. American Journal of Physical Anthropology 111:489-501.

Adams, D. C. 2016. Evaluating modularity in morphometric data: challenges with the RV coefficient and a new test measure. Methods Ecol. Evol. in press.

Adams, D. C. and R. N. Felice. 2014. Assessing trait covariation and morphological integration on phylogenies using evolutionary covariance matrices. PLoS ONE 9:e94335.

Akaike, H. 1973. Information theory as an extension of the maximum likelihood principle. Pp. 267-281 in B. N. Petrov, and F. Csaki, eds. Second International Symposium on Information Theory. Akademiai Kiado, Budapest.

Armbruster, W. S., C. Pelabon, G. H. Bolstad, and T. F. Hansen. 2014. Integrated phenotypes: understanding trait covariation in plants and animals. Phil Trans Roy Soc Lon B 369:20130245.

Armbruster, W. S., C. Pélabon, T. F. Hansen, and C. P. H. Mulder. 2004. Floral integration, modularity, and accuracy: distinguishing complex adaptations from genetic constraints. Pp. 23-49 in M. Pigliucci, and K. Preston, eds. Phenotypic integration. Oxford University Press, Oxford.

Bell, E., B. Andres, and A. Goswami. 2011. Limb integration and dissociation in flying vertebrates: a comparison of pterosaurs, birds, and bats. J. Evol. Biol. 24:286-2599.

Bennett, C. V. and A. Goswami. 2011. Does reproductive strategy drive limb integration in marsupials and monotremes? Mammalian Biology 76:79-83.

Bookstein, F. L. and P. Mitteroecker. 2014. Comparing covariance matrices by relative eigenanalysis, with applications to organismal biology. Evol. Biol. 41:336-350.

Burnham, K. P. and D. R. Anderson. 2002. Model Selection and Multimodel Inference: A Practical Information-Theoretic Approach. Springer, New York.

Burnham, K. P. and D. R. Anderson. 2004. Multimodel inference: Understanding AIC and BIC in model selection. Sociological Methods and Research 33:261-304.

Cheverud, J. M. 1982. Phenotypic, Genetic, and Environmental Morphological Integration in the Cranium. Evolution 36:499-516.

Cheverud, J. M. 1989. A comparative analysis of morphological variation patterns in the Papionines. Evolution 43:1737-1747.

Cheverud, J. M. 1995a. Morphological integration in the saddle-back tamarin (Saguinus fuscicollis) cranium. Am. Nat. 145:63-89.

Cheverud, J. M. 1995b. Morphological integration in the saddle-back tamarin (Saguinus fuscicollis) cranium. Am. Nat. 145:63-89.

Cheverud, J. M. 1996. Developmental integration and the evolution of pleiotropy. Am. Zool. 36:44-50.

Conner, J. K., I. A. Cooper, R. J. L. Rosa, S. G. Perez, and A. M. Royer. 2014. Patterns of phenotypic correlations among morphological traits in plants and animals. Phil Trans Roy Soc Lon B 369:20130246.

De Leeuw, J. 1983. Models and methods for the analysis of correlation coefficients. Journal of Econometrics 22:113-137.

Drake, A. G. and C. P. Klingenberg. 2010. Large-scale diversification of skull shape in domestic dogs: disparity and modularity. Am. Nat. 175:289-301. 
Edwards, A. W. F. 1992. Likelihood: Expanded Edition. The Johns Hopkins University Press, Baltimore.

Feldman, G., A. Hayes, S. Kumar, J. Greeson, and J.-P. Laurenceau. 2007. Mindfulness and emotion regulation: The development and initial validation of the Cognitive and Affective Mindfulness Scale-Revised (CAMS-R). Journal of Psychopathology and Behavioral Assessment 29:177-190.

Fruciano, C., P. Franchini, and A. Meyer. 2013. Resampling-based approaches to study variation in morphological modularity. PLoS ONE 8:e69376.

Gerber, S. 2013. On the relationship between the macroevolutionary trajectories of morphological integration and morphological disparity. PLoS ONE 8:e63913.

Gerber, S. and M. J. Hopkins. 2011. Mosaic heterochrony and evolutionary modularity: the trilobite genus Zacanthopsis as a case study. Evolution 65:3241-3252.

Goswami, A. 2006a. Cranial modularity shifts during mammalian evolution. Am. Nat. 168:270280.

Goswami, A. 2006b. Morphological integration in the carnivoran skull. Evolution 60:169-183.

Goswami, A. 2007. Phylogeny, diet, and cranial integration in australodelphian marsupials. PLoS One 2:e995.

Goswami, A., W. J. Binder, J. A. Meachen, and F. R. O'Keefe. 2015. The fossil record of phenotypic integration and modularity: a deep-time perspective on developmental and evolutionary dynamics. Proc. Natl. Acad. Sci. U. S. A. 112:4891-4896.

Goswami, A. and P. D. Polly. 2010. Methods for studying morphological integration and modularity. Pp. 213-243 in J. Alroy, and E. G. Hunt, eds. Quantitative Methods in Paleobiology. Paleontological Society Special Publications.

Goswami, A., J. B. Smaers, C. Soligo, and P. D. Polly. 2014. The macroevolutionary consequences of phenotypic integration: from development to deep time. Phil Trans Roy Soc Lon B 369:20130254.

Goswami, A., V. Weisbecker, and M. R. Sanchez-Villagra. 2009. Developmental Modularity and the Marsupial-Placental Dichotomy. J. Exp. Zool. Part B 312B:186-195.

Hallgrimsson, B., H. Jamniczky, N. M. Young, C. Rolian, T. E. Parsons, J. C. Boughner, and R. S. Marcucio. 2009. Deciphering the palimpsest: studying the relationship between morphological integration and phenotypic covariation. Evol. Biol. 36:355-376.

Hallgrimsson, B., K. Willmore, C. Dorval, and D. M. L. Cooper. 2004. Craniofacial variability and modularity in macaques and mice. J. Exp. Zool. Part B 302B:207-225.

Hurvich, C. M. and C.-L. Tsai. 1989. Regression and time series model selection in small samples. Biometrika 76:297-307.

Klingenberg, C. P. 2008. Morphological integration and developmental modularity. Annual Review of Ecology, Evolution, and Systematics 39:115-132.

Klingenberg, C. P. 2009. Morphometric integration and modularity in configurations of landmarks: tools for evaluating a prior hypotheses. Evol. Dev. 11:405-421.

Klingenberg, C. P. 2013. Cranial integration and modularity: insights into evolution and development from morphometric data. Hystrix 24:43-58.

Klingenberg, C. P. 2014. Studying morphological integration and modularity at multiple levels: concepts and analysis. Phil Trans Roy Soc Lon B 369:in press.

Klingenberg, C. P. and J. Marugan-Lobon. 2013. Evolutionary covariation in geometric morphometric data: analyzing integration, modularity and allometry in a phylogenetic context. Syst. Biol. 62:591-610. 
LeBel, E. P. and B. Gawronski. 2009. How to find what's in a name: Scrutinizing the optimality of five scoring algorithms for the name-letter task. European Journal of Personality 23:85-106.

Marquez, E. J. 2008. A statistical framework for testing modularity in multidimensional data. Evolution 62:2688-2708.

Marroig, G. and J. M. Cheverud. 2001. A comparison of phenotypic variation and covariation patterns and the role of phylogeny, ecology, and ontogeny during cranial evolution of New World monkeys. Evolution 55:2576-2600.

Marroig, G., L. Shirai, A. Porto, F. B. de Oliveira, and V. De Conto. 2009. The evolution of modularity in the mammalian skull II: evolutionary consequences. Evol. Biol. 36:136148.

Maxwell, E. E. and T. A. Dececchi. 2012. Ontogenetic and stratigraphic influence on observed phenotypic integration in the limb skeleton of a fossil tetrapod. Paleobiology 39:123-134.

Meloro, C. and G. J. Slater. 2012. Covariation in the skull modules of cats: the challenge of growing saber-like canines. J. Vert. Paleontol. 32:677-685.

Olson, E. C. and R. L. Miller. 1951. A mathematical model applied to the evolution of species. Evolution 5:325-338.

Olson, E. C. and R. L. Miller. 1958. Morphological Integration. University of Chicago Press, Chicago

Pavlicev, M., J. M. Cheverud, and G. P. Wagner. 2009. Measuring morphological integration using eigenvalue variance. Evol. Biol. 36:157-170.

Porto, A., F. B. de Oliveira, L. Shirai, V. De Conto, and G. Marroig. 2009. The evolution of modularity in the mammalian skull I: morphological integration patterns and magnitudes. Evol. Biol. 36:118-135.

Rohlf, F. J. and M. Corti. 2000. Use of two-block partial least-squares to study covariation in shape. Syst. Biol. 49:740-753.

Royall, R. M. 1997. Statistical Evidence: A Likelihood Paradigm. Chapman and Hall, New York.

Sears, K. E., C. Doroba, X. Cao, D. Xie, and S. Zhong. 2012. Molecluar determinants of marsupial integration and constraint in R. J. Asher, and J. Mueller, eds. From clone to bone: the synergy of morphological and molecular tools in palaeobiology. Cambridge University Press, Cambridge.

Sokal, R. R. and F. J. Rohlf. 1995. Biometry. W. H. Freeman, New York.

Steiger, J. H. 1980a. Testing pattern hypotheses on correlation matricies: alternative statistics and some empirical results. Multivariate Behavioral Research 15:335-352.

Steiger, J. H. 1980b. Tests for comparing elements of a correlation matrix. Psychological Bulletin 87:245-251.

Wager, T. D., D. J. Scott, and J.-K. Zubieta. 2007. Placebo effects on human $\mu$-opioid activity during pain. Proceedings of the National Academy of Sciences 104:11056-11061.

Wagner, P. J. 2000. Likelihood tests of hypothesized durations: determining and accommodating biasing factors. Paleobiology 26:431-449.

Webster, M. and M. L. Zelditch. 2011a. Evolutionary lability of integration in Cambrian ptychopariod trilobites. Evol. Biol. 38:144-162.

Webster, M. and M. L. Zelditch. 2011b. Modularity of a Cambrian ptychoparioid trilobite cranidium. Evol. Dev. 13:96-109. 
773 Young, N. M. and B. Hallgrimsson. 2005. Serial homology and the evolution of mammalian $774 \quad$ limb covariation structure. Evolution 59:2691-2704.

775 Young, R. W. 1959. The influence of cranial contents on postnatal growth of the skull in the rat. $776 \quad$ American Journal of Anatomy 105:383-415.

777 Zelditch, M. L. 1988. Ontogenetic variation in patterns of phenotypic integration in the $778 \quad$ laboratory rat. Evolution 42:28-41.

779 Zelditch, M. L., J. G. Mezey, H. D. Sheets, B. L. Lundrigan, and J. T. Garland. 2006.

780 Developmental regulation of skull morphology II: Ontogenetic dynamics of covariance.

$781 \quad$ Evol. Biol. 8:46-60.

782 Zelditch, M. L., A. R. Wood, and D. L. Swiderski. 2009. Building developmental integration into 783 functional systems: function-induced integration of mandibular shape. Evol. Biol. 36:7178487.

785

786 
787

788

789

790

791

792

793

794

795

796

797

798

799

800

801

802

803

804

805

806

807

\section{FIGURE CAPTIONS}

Figure 1. Schematic depiction of three alternative partitions of the macaque cranium. A) No modularity, with similar levels of correlation among all landmarks. B) Two modules, corresponding to facial and neurocranial regions. C) Six modules, corresponding approximately to Cheverud's model (1982). Colored circles indicate module associations. Solid lines indicate within-module correlations. Dotted lines indicate between-module correlations.

Figure 2. Schematic depiction of the four alternative parameterizations of a single six-module model structure. A) Basic structure of landmark associations in six modules, indicated by colours. The six modules may have either similar (B) or different (C) magnitudes of withinmodule correlations. The intermodule correlations may also be similar (D and F) or different (E and $\mathrm{G}$ ) among all pairs of modules. Each distinct estimated value of $\rho$ is counted as a parameter, along with one additional parameter for estimated variance. Solid lines indicate within-module correlations. Dashed lines indicate between-module correlations. Line colours indicate similar or different estimated values for $\rho$ (e.g., in B, the black lines indicate that all of the six modules have the same estimated within-module correlation).

Figure 3. Results of simulations demonstrating accuracy in model selection for different model structures (no modularity, two modules, or six modules), complexity (similar or different withinmodule correlations), and magnitudes of within-module correlations, modelled with varying standard deviations of A) $\sigma=0.01$ or B) $\sigma=0.05$. Stacked bars show percentage of simulations 
808 identifying: the correct model (green), an alternative parameterization of the same model

809 structure, i.e., a related model, with posterior probability $<0.50$ (dark blue), a related model with

810 posterior probability $>0.50$ (light blue), an unrelated model with posterior probability $<0.50$

811 (pink), or an unrelated model with posterior probability $>0.50$ (red). Simulated mean within-

812 module correlations, or all correlations for no modularity models, are indicated on the x-axis.

813100 simulations were run for each model, resulting in a total of 4200 simulations. Results show

814 that this method is highly accurate at identifying the correct model structure, except where higher

815 standard deviations are combined with extremely high correlations and simple model structures

816 (no modularity, in particular).

817 


\section{TABLES}

Table 1: Model descriptions and parameterizations for the 31 model structures explored in this study. Base models structures follow the allocation of landmark variables in Table S1. Model parameters are a sum of the number of estimated correlations within modules and across modules, plus one (for the estimate of the variance of the population correlation).

$\begin{array}{ccccc}\text { Model ID } & \text { Base Model Structure } & \text { \# Modules } & \text { Model description } \\ 1 & \text { No Modules } & 0 & 1 \rho \text { for all correlations } \\ 2 & \text { Neurocranial/Facial model } & 2 & 1 \text { within module } \rho \text { for both modules, } 1 \text { between-module } \rho \\ 3 & \text { Neurocranial/Facial model } & 2 & 2 \text { within-module } \rho \text { 's and } 1 \text { between-module } \rho \\ 4 & \text { Cheverud model } & 6 & 1 \text { within-module } \rho \text { and } 1 \text { between-module } \rho \\ 5 & \text { Cheverud model } & 6 & \text { Separate within-module } \rho \text { 's and } 1 \text { between-module } \rho \\ 6 & \text { Cheverud model } & 6 & 1 \text { within-module } \rho \text { and separate between-module } \rho \text { 's } \\ 7 & \text { Cheverud model } & 6 & \text { Separate within-module } \rho \text { 's and separate between-module } \rho \text { 's } \\ 8 & \text { Goswami model } & 6 & 1 \text { within-module } \rho \text { and } 1 \text { between-module } \rho \\ 9 & \text { Goswami model } & 6 & \text { Separate within-module } \rho \text { 's and } 1 \text { between-module } \rho & 3 \\ 10 & \text { Goswami model } & 6 & 1 \text { within-module } \rho \text { and separate between-module } \rho \text { 's } \\ 11 & \text { Goswami model } & 6 & \text { Separate within-module } \rho \text { 's and separate between-module } \rho \text { 's } & 8 \\ 12 & \text { Cheverud/Goswami } & 8 & 1 \text { within-module } \rho \text { and } 1 \text { between-module } \rho\end{array}$


combined model

\section{Cheverud/Goswami}

13 combined model

14

\section{Cheverud/Goswami}

combined model

Cheverud/Goswami

$$
\text { combined model }
$$

Tissue Origin model

Tissue Origin model

Tissue Origin model

Tissue Origin mode

Cheverud-based

"monotreme" model

Cheverud-based

"monotreme" model

Cheverud-based

"monotreme" model

Cheverud-based
Separate within-module $\rho$ 's and 1 between-module $\rho$

10

Separate within-module $\rho$ 's and separate between-module $\rho$ 's

1 within-module $\rho$ and 1 between-module $\rho$

1 within-module $\rho$ and separate between-module $\rho$ 's

Separate within-module $\rho$ and 1 between-module $\rho$ 's

Separate within-module $\rho$ and separate between-module $\rho$ 's

1 within-module $\rho$ (for modules 1,2 , and 6 only), 1 pooled between-module and unintegrated $\rho$

1 within-module $\rho$ (for modules 1,2 , and 6 only), 1 betweenmodule $\rho$, and 1 unintegrated $\rho$

Separate within-module $\rho$ 's (for modules 1, 2, and 6 only), 1 pooled between-module and unintegrated $\rho$

Separate within-module $\rho$ 's (for modules 1, 2, and 6 only), 1 
"monotreme" model

Cheverud-based

24

"monotreme" model

25

Cheverud-based

"monotreme" model

Goswami-based

"monotreme" model

Goswami-based

27

"monotreme" model

Goswami-based

"monotreme" model

Goswami-based

"monotreme" model

Goswami-based

30

“monotreme" model

Goswami-based

"monotreme" model between-module $\rho$, and 1 unintegrated $\rho$

1 within-module $\rho$ (for modules 1,2 , and 6 only), separate between-module $\rho$ 's, and 1 unintegrated $\rho$

Separate within-module $\rho$ 's (for modules 1, 2, and 6 only), separate between-module p's, and 1 unintegrated $\rho$

1 within-module $\rho$ (for modules 1,2 , and 6 only), 1 pooled between-module and unintegrated $\rho$

1 within-module $\rho$ (for modules 1,2 , and 6 only), 1 betweenmodule $\rho$, and 1 unintegrated $\rho$

Separate within-module $\rho$ 's (for modules 1,2 , and 6 only), 1 pooled between-module and unintegrated $\rho$

Separate within-module $\rho$ 's (for modules 1,2 , and 6 only), 1 between-module $\rho$, and 1 unintegrated $\rho$

1 within-module $\rho$ (for modules 1,2 , and 6 only), separate between-module $\rho$ 's, and 1 unintegrated $\rho$

Separate within-module $\rho$ 's (for modules 1, 2, and 6 only), separate between-module p's, and 1 unintegrated $\rho$ 
Table 2: Results for the Sub-Adult (M2 erupted) data set $(\mathrm{n}=48)$ using congruence coefficients. Model parameters, raw loglikelihood fits for each tested model, $\mathrm{AIC}_{\mathrm{c}}$ and $\Delta \mathrm{AIC} \mathrm{C}_{\mathrm{c}}$ scores are provided. Model log-likelihoods and the model posterior probability are also shown. Sample size used to calculate $\mathrm{AIC}_{\mathrm{c}}$ was 1830 . See methods for details. Model ID's correspond to the numbering in Table 1. The optimal model in the set of evaluated models is highlighted in bold italics.

\begin{tabular}{|c|c|c|c|c|c|c|}
\hline Model ID & $\mathbf{K}$ & $\log L$ & $\mathrm{AIC}_{\mathrm{c}}$ & $\Delta \mathrm{AIC}_{\mathrm{c}}$ & Model LogL & Model Post. Prob. \\
\hline 1 & 2 & 2078.86 & -4153.72 & 916.21 & $1.11 \mathrm{E}-199$ & $1.11 \mathrm{E}-199$ \\
\hline 2 & 3 & 2134.49 & -4262.97 & 806.96 & $5.89 \mathrm{E}-176$ & $5.89 \mathrm{E}-176$ \\
\hline 3 & 4 & 2147.54 & -4287.06 & 782.88 & $1.00 \mathrm{E}-170$ & $1.00 \mathrm{E}-170$ \\
\hline 4 & 3 & 2219.34 & -4432.67 & 637.26 & 4.17E-139 & $4.17 \mathrm{E}-139$ \\
\hline 5 & 8 & 2380.83 & -4745.58 & 324.35 & $3.69 \mathrm{E}-71$ & 3.69E-71 \\
\hline 6 & 17 & 2395.76 & -4757.18 & 312.75 & $1.22 \mathrm{E}-68$ & $1.22 \mathrm{E}-68$ \\
\hline 7 & 22 & 2557.25 & -5069.93 & 0.00 & 1.00 & 1.000 \\
\hline 8 & 3 & 2153.94 & -4301.87 & 768.06 & $1.65 \mathrm{E}-167$ & $1.65 \mathrm{E}-167$ \\
\hline 9 & 8 & 2226.56 & -4437.03 & 632.90 & $3.69 \mathrm{E}-138$ & $3.69 \mathrm{E}-138$ \\
\hline 10 & 17 & 2257.63 & -4480.93 & 589.01 & $1.26 \mathrm{E}-128$ & $1.26 \mathrm{E}-128$ \\
\hline 11 & 22 & 2330.25 & -4615.93 & 454.00 & $2.60 \mathrm{E}-99$ & 2.60E-99 \\
\hline 12 & 3 & 2172.35 & -4338.69 & 731.24 & $1.63 \mathrm{E}-159$ & $1.63 \mathrm{E}-159$ \\
\hline 13 & 10 & 2246.04 & -4471.95 & 597.98 & $1.41 \mathrm{E}-130$ & $1.41 \mathrm{E}-130$ \\
\hline
\end{tabular}




\begin{tabular}{|c|c|c|c|c|c|c|}
\hline 14 & 30 & 2417.44 & -4773.85 & 296.09 & $5.07 \mathrm{E}-65$ & $5.07 \mathrm{E}-65$ \\
\hline 15 & 37 & 2491.12 & -4906.68 & 163.26 & $3.54 \mathrm{E}-36$ & $3.54 \mathrm{E}-36$ \\
\hline 16 & 3 & 2079.47 & -4152.93 & 917.00 & $7.50 \mathrm{E}-200$ & $7.50 \mathrm{E}-200$ \\
\hline 17 & 5 & 2214.56 & -4419.08 & 650.85 & $4.67 \mathrm{E}-142$ & 4.67E-142 \\
\hline 18 & 5 & 2109.73 & -4209.43 & 860.51 & $1.39 \mathrm{E}-187$ & $1.39 \mathrm{E}-187$ \\
\hline 19 & 7 & 2244.82 & -4475.57 & 594.36 & $8.62 \mathrm{E}-130$ & $8.62 \mathrm{E}-130$ \\
\hline 20 & 3 & 2262.47 & -4518.93 & 551.01 & $2.24 \mathrm{E}-120$ & $2.24 \mathrm{E}-120$ \\
\hline 21 & 4 & 2265.54 & -4523.05 & 546.88 & $1.76 \mathrm{E}-119$ & $1.76 \mathrm{E}-119$ \\
\hline 22 & 5 & 2324.39 & -4638.75 & 431.18 & 2.34E-94 & 2.34E-94 \\
\hline 23 & 6 & 2327.46 & -4642.87 & 427.06 & $1.84 \mathrm{E}-93$ & $1.84 \mathrm{E}-93$ \\
\hline 24 & 6 & 2286.11 & -4560.17 & 509.76 & $2.03 \mathrm{E}-111$ & $2.03 \mathrm{E}-111$ \\
\hline 25 & 8 & 2348.03 & -4679.99 & 389.95 & $2.11 \mathrm{E}-85$ & $2.11 \mathrm{E}-85$ \\
\hline 26 & 3 & 2181.12 & -4356.23 & 713.70 & $1.05 \mathrm{E}-155$ & $1.05 \mathrm{E}-155$ \\
\hline 27 & 4 & 2181.12 & -4354.23 & 715.71 & $3.85 \mathrm{E}-156$ & $3.85 \mathrm{E}-156$ \\
\hline 28 & 5 & 2204.15 & -4398.27 & 671.66 & $1.42 \mathrm{E}-146$ & $1.42 \mathrm{E}-146$ \\
\hline 29 & 6 & 2204.15 & -4396.26 & 673.67 & $5.17 \mathrm{E}-147$ & 5.17E-147 \\
\hline 30 & 6 & 2195.90 & -4379.76 & 690.18 & $1.35 \mathrm{E}-150$ & $1.35 \mathrm{E}-150$ \\
\hline 31 & 8 & 2218.93 & -4421.78 & 648.15 & $1.80 \mathrm{E}-141$ & $1.80 \mathrm{E}-141$ \\
\hline
\end{tabular}


Table 3: Optimal values of $\rho$ within the six modules and for the 15 inter-module correlations estimated in Model 7 for each of the macaque data sets partitioned by ontogenetic stage.

\begin{tabular}{|c|c|c|c|c|c|}
\hline & $\begin{array}{c}\text { Adult } \\
\text { Females }\end{array}$ & $\begin{array}{l}\text { Adult } \\
\text { Males }\end{array}$ & $\begin{array}{l}\text { Sub-Adult } \\
\text { (M2 erupted) }\end{array}$ & $\begin{array}{c}\text { Juvenile } \\
\text { (M1 erupted) }\end{array}$ & $\begin{array}{c}\text { Infant } \\
\text { (Deciduous only) }\end{array}$ \\
\hline Module 1 & 0.43 & 0.46 & 0.43 & 0.44 & 0.55 \\
\hline Module 2 & 0.77 & 0.77 & 0.81 & 0.76 & 0.67 \\
\hline Module 3 & 0.24 & 0.35 & 0.40 & 0.19 & 0.22 \\
\hline Module 4 & 0.15 & 0.18 & 0.14 & 0.16 & 0.15 \\
\hline Module 5 & 0.12 & 0.23 & 0.14 & 0.17 & 0.23 \\
\hline Module 6 & 0.28 & 0.29 & 0.30 & 0.30 & 0.28 \\
\hline M1 to M2 & 0.10 & 0.13 & 0.13 & 0.13 & 0.13 \\
\hline M1 to M3 & 0.22 & 0.29 & 0.35 & 0.21 & 0.31 \\
\hline M1 to M4 & 0.18 & 0.22 & 0.14 & 0.14 & 0.20 \\
\hline M1 to M5 & 0.21 & 0.21 & 0.22 & 0.22 & 0.29 \\
\hline M1 to M6 & 0.19 & 0.17 & 0.22 & 0.20 & 0.28 \\
\hline M2 to M3 & 0.13 & 0.22 & 0.08 & 0.08 & 0.12 \\
\hline M2 to M4 & 0.14 & 0.08 & 0.12 & 0.08 & 0.14 \\
\hline M2 to M5 & 0.07 & 0.09 & 0.10 & 0.13 & 0.10 \\
\hline
\end{tabular}




$\begin{array}{llllll}\text { M2 to M6 } & 0.12 & 0.27 & 0.08 & 0.17 & 0.08 \\ \text { M3 to M4 } & 0.11 & 0.15 & 0.11 & 0.11 & 0.13 \\ \text { M3 to M5 } & 0.16 & 0.12 & 0.16 & 0.09 & 0.16 \\ \text { M3 to M6 } & 0.11 & 0.12 & 0.15 & 0.10 & 0.14 \\ \text { M4 to M5 } & 0.14 & 0.15 & 0.11 & 0.12 & 0.13 \\ \text { M4 to M6 } & 0.13 & 0.12 & 0.11 & 0.11 & 0.11 \\ \text { M5 to M6 } & 0.17 & 0.17 & 0.14 & 0.16 & 0.15\end{array}$


$80 \quad 0^{\circ}$

$$
\begin{aligned}
& \text { A. No modularity } \\
& \begin{array}{llll}
0 & 0 & 0 & 00 \\
0 & 0 & 0 & 00
\end{array}
\end{aligned}
$$
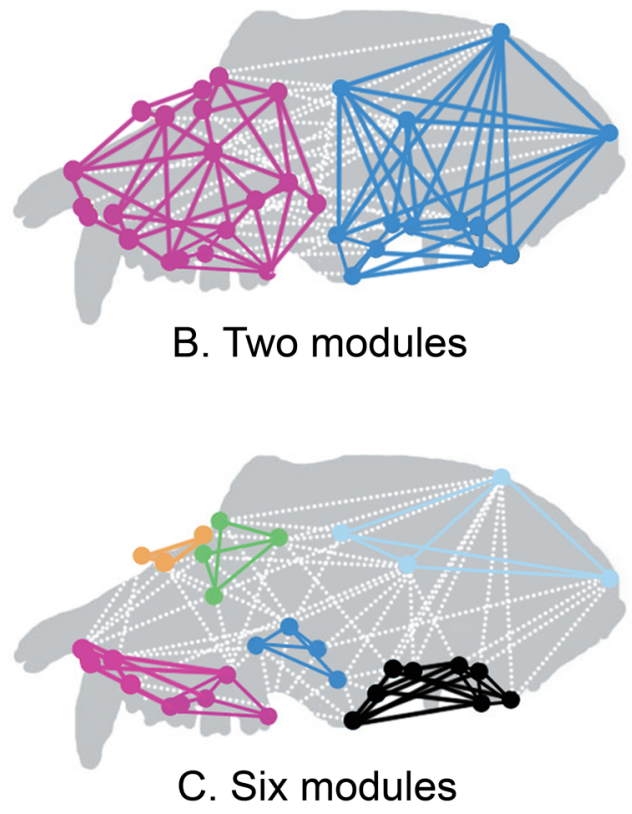


\section{Page 45 of 46}

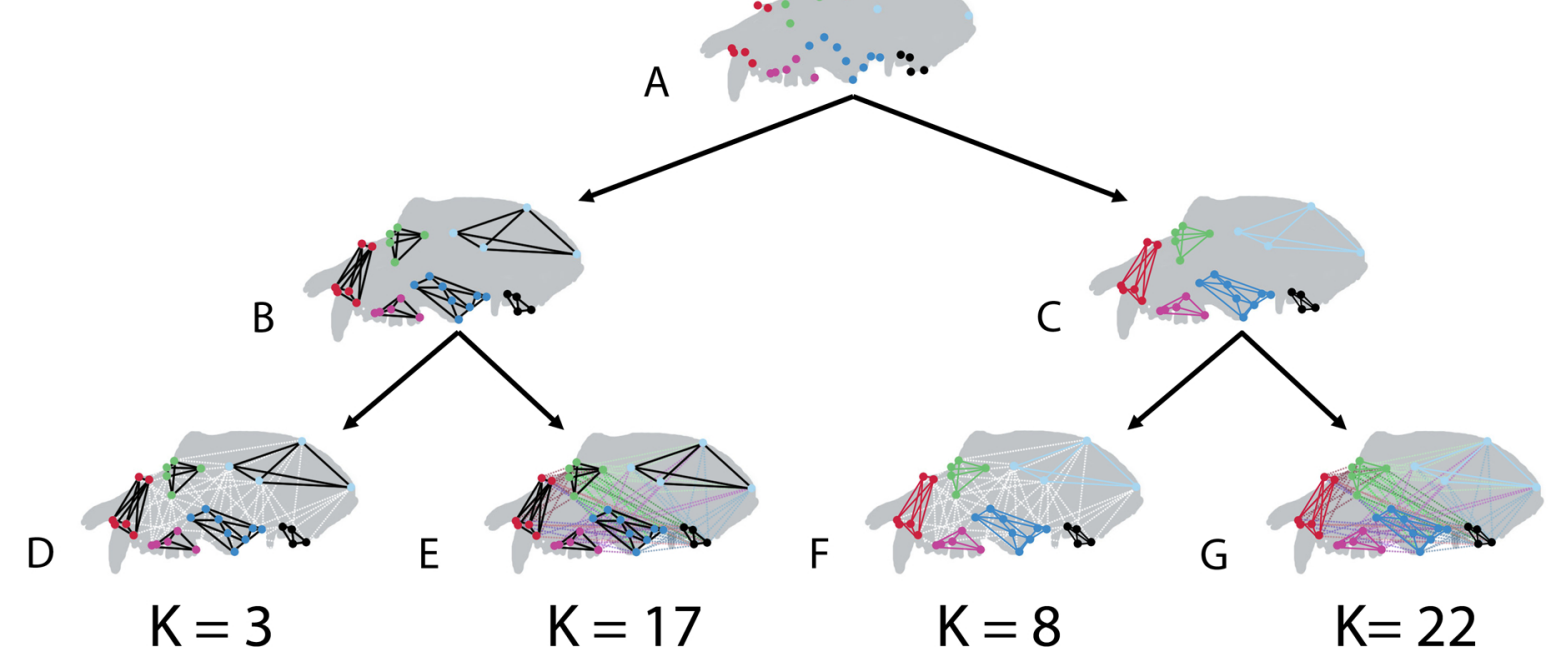


A) $\sigma=0.01$

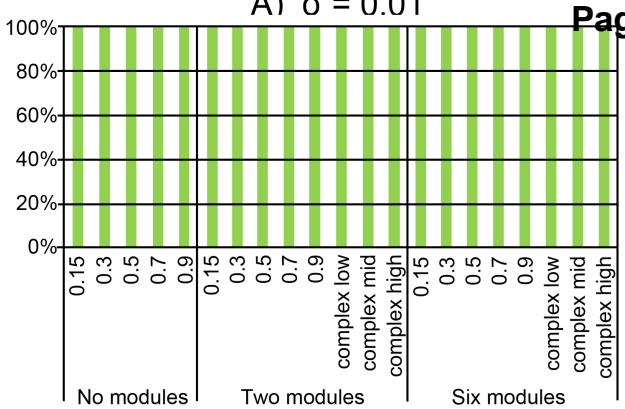

U Unrelated PP>0.5

Unrelated $\mathrm{PP}<0.5$

Related PP>0.5

- Related $\mathrm{PP}<0.5$

Correct

B) $\sigma=0.05$

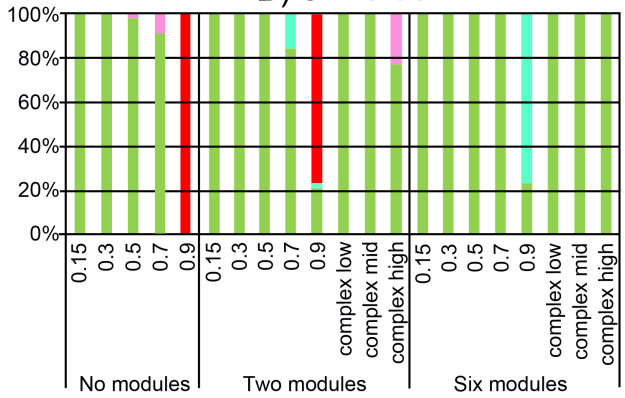

Unrelated PP>0.5

Unrelated $\mathrm{PP}<0.5$

Related PP>0.5

Related $\mathrm{PP}<0.5$

Correct 